\title{
Segmental and complementary expression of L-serine biosynthetic enzyme 3-phosphoglycerate dehydrogenase and neutral amino acid transporter ASCT1 in the mouse kidney
}

\author{
Chihiro Takasaki, Eriko Miura and Masahiko Watanabe \\ Department of Anatomy, Hokkaido University School of Medicine, Sapporo 060-8638, Japan \\ (Received 25 December 2006; and accepted 15 January 2007)
}

\begin{abstract}
3-Phosphoglycerate dehydrogenase (Phgdh) is the initial step enzyme in the phosphorylated pathway of L-serine biosynthesis. We have previously revealed in the brain that Phgdh is preferentially expressed in glial cells, but not in neurons, and that glia-borne L-serine exerts strong neurotrophic actions to neuronal survive, differentiation, and development. To investigate whether such an L-serine-meditated intercellular relationship is constructed in peripheral organs and tissues, we examined the kidney, which is one of the organs with the highest expression of Phgdh mRNA in the body. We found that Phgdh was distributed highly in the renal papilla and inner layer of the outer zone and moderately in the cortex, whereas it was almost negative in the outer layer of the outer zone. This heterogeneous distribution was due to selective expression in distinct tubular segments, i.e., the Bowman's capsule, proximal tubule, and thin limbs of the Henle's loop. Interestingly, neutral amino acid transporter ASCT1, which preferentially transports alanine, serine, cysteine, and threonine, was selectively expressed in Phgdh-negative tubular segments, i.e., the distal tubule and collecting duct. Therefore, either Phgdh or ASCT1 is provided to each segment of renal tubules, suggesting that metabolic interplay mediated by L-serine biosynthesis and supply may exist in the kidney too.
\end{abstract}

L-Serine is classified as a non-essential amino acid, since it can be synthesized sufficiently in human and animal bodies and the nitrogen balance is maintained without dietary intake (19). This amino acid serves not only as a building block of proteins, but also as a precursor for other amino acids (L-cysteine, glycine, D-serine), phosphatidylserine, sphingolipids, and nucleotides. 3-Phosphoglycerate dehydrogenase (Phgdh or 3PGDH, EC 1.1.1.95) is the initial step enzyme in the phosphorylated pathway of L-serine biosynthesis, using 3-phosphoglycerate, an intermediate of the glycolytic pathway, as a starting materi-

Address correspondence to: Dr. Masahiko Watanabe Department of Anatomy, Hokkaido University School of Medicine, Sapporo 060-8638, Japan

Tel: +81-11-706-5032, Fax: +81-11-706-5031

E-mail: watamasa@med.hokudai.ac.jp al (11). Phgdh is expressed in various organs and tissues, and is significantly upregulated in proliferating, differentiating, and neoplastic tissues $(5,12$, 22). The fundamental importance of L-serine biosynthesis in vivo is appreciated from severe neurological symptoms of patients with inherited Phgdh deficiency, including congenital microcephaly, dysmyelination, intractable seizures, and psychomotor retardation (6). Furthermore, targeted disruption of the Phgdh gene in mice results in embryonic lethality at embryonic day 13.5 accompanied by striking defects and retardation of brain development (25).

L-Serine plays as a glia-derived neurotrophic factor in the brain $(9,13)$. When astrocyte-conditioned medium or L-serine is supplied to cultured neurons, survival, dendritogenesis, synaptogenesis, and maturation of electrophysiological properties of the cultured neurons are greatly promoted, showing much 
greater potency than that by neurotrophins (9). Why these experimental treatments manifest neurotrophic actions is now understood by the fact that Phgdh is expressed primarily by astrocytes, olfactory ensheathing glia, and Schwann cells, whereas neurons lack its expression $(9,22,24)$. Furthermore, these Phgdh-expressing glial cells also express neutral amino acid transporter ASCT1, which mediates obligatory exchange of alanine, serine, cysteine, and threonine (17). Based on these findings, we have concluded that L-serine biosynthesis in particular glial cells and its supply to nearby neurons constitute a novel neuron-glial relationship for normal brain development and function (10).

In the present study, we tested whether such an intercellular relationship based on the L-serine metabolism is built up in peripheral organs and tissues as well. For this purpose, we selected the kidney, because this is one of the organs with the highest expression of Phgdh mRNA $(1,12)$. Here we show that, similarly to the case in the brain, cellular expression of Phgdh is not ubiquitous but rather preferential to particular tubular segments. Unlike the case in the brain, Phgdh and ASCT1 are expressed in distinct tubular segments.

\section{MATERIALS AND METHODS}

Animals and section preparation. C57BL/6J mice at the adult stage (2-4 months of age) were used in the present study. Under deep pentobarbital anesthesia $(100 \mathrm{mg} / \mathrm{kg}$ of body weight, i.p.), the kidney for in situ hybridization was freshly obtained and frozen immediately with powdered dry ice. Frozen sections were prepared on a cryostat $(20 \mu \mathrm{m}$ in thickness; CM1900; Leica, Nussloch, Germany), mounted on silane-coated glass slides (Muto-Glass, Tokyo, Japan) and air-dried. For light microscopic immunohistochemistry, anesthetized mice were fixed transcardially with $4 \%$ paraformaldehyde in $0.1 \mathrm{M}$ sodium phosphate buffer ( $\mathrm{PB}, \mathrm{pH}$ 7.2), and processed for paraffin sectioning with a sliding microtome $(4 \mu \mathrm{m}$; SM2000R, Leica) or for cryostat sections $(50 \mu \mathrm{m}$; CM1900, Leica). For electron microscopic immunohistochemistry, 4\% paraformaldehyde $/ 0.1 \%$ glutaraldehyde in $0.1 \mathrm{M}$ PB was used for transcardial perfusion, and microslicer sections were prepared (50 $\mu \mathrm{m}$; VT1000S, Leica). Mice were treated according to the guidelines for the care and use of laboratory animals of Hokkaido University School of Medicine.

In situ hybridization. Three antisense oligonucle- otides were synthesized as probes for mouse Phgdh mRNA. Sequences of probes was 5'-TGCAGCC CTCCATCTTGCAGGATCTTCCGGCAGCAG GGGTCCAGG-3', 5'-GAGCCAGCCCAGGCGTG CATCAGCGTGCCCATTGCTTCTGCCAGA-3', and 5'-AGGGAGGACAGGCCCATGACGTGC CAGGGCTCTCCGTCAGACACC-3' correspond to nucleotide residues 121-165, 1081-1125, and 15761675 of mouse Phgdh cDNA (GenBank accession number BC110673). They were processed for probe labeling with $\left[{ }^{33} \mathrm{P}\right] \mathrm{dATP}$, prehybridization, hybridization, washing, and autoradiography to X-ray films, as reported previously (22). After confirming the same pattern of hybridization by individual probes, three probes were mixed in order to raise the gain of signals.

Immunohistochemistry. All immunohistochemical incubations were performed at room temperature. For light microscopic immunoperoxidase, paraffin sections were incubated with $10 \%$ normal goat serum for $30 \mathrm{~min}$, rabbit anti-Phgdh antibody $(1 \mu \mathrm{g} /$ $\mathrm{mL}$ ) (22) overnight, biotinylated goat anti-rabbit IgG for $2 \mathrm{~h}$, and streptavidin-peroxidase complex for $1 \mathrm{~h}$, using a Histofine SAB-PO(R) kit (Nichirei Corporation, Tokyo, Japan). Immunoreaction was visualized with 3,3'-diaminobenzidine (DAB). Some sections were counterstained with hematoxylin. For immunofluorescence, sections were immunoreacted overnight with rabbit or guinea pig anti-Phgdh antibody $(1 \mu \mathrm{g} / \mathrm{mL})$ in combination with rabbit anti-aquaporin 1 (AQP1) antibody $(2 \mu \mathrm{g} / \mathrm{mL}$; CHEMICON International, Temecula, CA, USA), goat anti-aquaporin 2 (AQP2) antibody $(1 \mu \mathrm{g} / \mathrm{mL}$; Santa Cruz Biotechnology, Santa Cruz, CA, USA), rabbit anti-chloride channel-K (CLC-K) antibody $(1 \mu \mathrm{g} / \mathrm{mL}$; Alamone Laboratories, Jerusalem, Israel), guinea pig anti-ASCT1 antibody $(1 \mu \mathrm{g} / \mathrm{mL})(17)$, or goat anti-calbindin antibody $(1 \mu \mathrm{g} / \mathrm{mL})(15)$. They were incubated for $2 \mathrm{~h}$ with a mixture of Alexa Fluor-488 and Cy3-conjugated secondary antibody (Invitrogen, Carlsbad, CA, USA; Jackson ImmunoResearch, West Grove, PA, USA). Some sections were counterstained with $1 \mu \mathrm{M}$ TOTO3 (Molecular Probes, Eugene, OR, USA) for $10 \mathrm{~min}$. Phosphatebuffered saline (PBS) containing $0.1 \%$ Tween 20 was used for antibody diluent and washing buffer. Photographs were taken by a light microscope (AX-70; Olympus Optical, Tokyo, Japan) equipped with a digital camera (DP70; Olympus Optical) or by a confocal laser scanning microscope (FV1000; Olympus Optical). We tested the specificity of immunohistochemistry by blank labeling when the pri- 
mary antibody was omitted.

For silver-enhanced immunogold labeling, microslicer sections were incubated successively with $5 \%$ bovine serum albumin in PBS containing $0.002 \%$ saponin for $30 \mathrm{~min}$, rabbit anti-Phgdh antibody $(1 \mu \mathrm{g} /$ $\mathrm{mL}$ ) overnight, and anti-rabbit $\mathrm{IgG}$ conjugated to $1.4 \mathrm{~nm}$ gold particles $(1: 200$; Nanoprobes, Stony Brook, NY, USA) overnight. Following postfixation with $1 \%$ glutaraldehyde in PB for 30 min and silver enhancement (HQ Silver Kit, Nanoprobes), sections were osmificated with $1 \%$ osmium tetroxide for $1.5 \mathrm{~h}$ and stained with $1 \%$ uranyl acetate for $30 \mathrm{~min}$. All sections were dehydrated using graded alcohols and embedded in Epon 812. Ultrathin sections were prepared with an ULTRACUT ultramicrotome (Leica, Wien, Austria), and photographs were taken with an H7100 electron microscope (Hitachi, Tokyo, Japan).

Western blot analysis. The kidney was homogenized using a Potter homogenizer with 15 strokes at $1000 \mathrm{rpm}$ in 10 volumes of ice-cold homogenizer buffer containing $0.32 \mathrm{M}$ sucrose, $1 \mathrm{mM}$ EDTA, $0.4 \mathrm{mM}$ EGTA, $10 \mathrm{mM}$ Tris- $\mathrm{HCl}$ (pH 7.2), and $0.4 \mathrm{mM}$ phenylmethylsulfonyl fluoride. The protein concentration was determined by the Lowry's method. The homogenates were denatured by $50 \mathrm{mM}( \pm)$ dithiothreitol at $65^{\circ} \mathrm{C}$ for $15 \mathrm{~min}$. Proteins $(15 \mu \mathrm{g} /$ lane) were separated by SDS-polyacrylamide gel electrophoresis, and electroblotted onto nitrocellulose membranes (BioTraceNT; Pall Gelman Laboratory, Ann Arbor, MI, USA). After blocking with 5\% skimmed milk for $30 \mathrm{~min}$, membranes were incubated for $2 \mathrm{~h}$ with Phgdh antibody $(1 \mu \mathrm{g} / \mathrm{mL})$. Tris- buffered saline, $\mathrm{pH} 7.5$, containing $0.1 \%$ Triton $\mathrm{X}-100$ was used for antibody diluent and washing buffer. Immunoreaction was visualized with the ECL chemiluminescence detection system (Amersham Biosciences).

\section{RESULTS}

\section{Phgdh mRNA expression in adult kidney}

By in situ hybridization with ${ }^{33} \mathrm{P}$-labeled antisense probes, expression of Phgdh mRNA was examined in the adult mouse kidney (Fig. 1). Prominent expression was detected in the renal papilla (Fig. 1A). In addition, clusters of low signals were sparsely distributed in the renal cortex and in the inner layer of the outer zone, whereas its outer layer lacked the signals. The specificity of hybridizing signals was tested by control hybridization, in which signals disappeared almost completely when hybridization was carried out in the presence of an excess amount of cold (unlabeled) probes (Fig. 1B).

\section{Specificity of Phgdh antibody and immunohisto- chemistry}

The specificity of Phgdh antibody was tested by immunoblot (Fig. 2). A single protein band at $60 \mathrm{kDa}$ was detected in homogenates prepared from the kidney (Fig. 2, right). The size of Phgdh in the kidney was similar to that in the brain (Fig. 2, left). Tissue contents of Phgdh was higher in the kidney than in the brain.

Immunoperoxidase staining revealed distinct distribution of Phgdh in the adult kidney (Fig. 3). Phg-
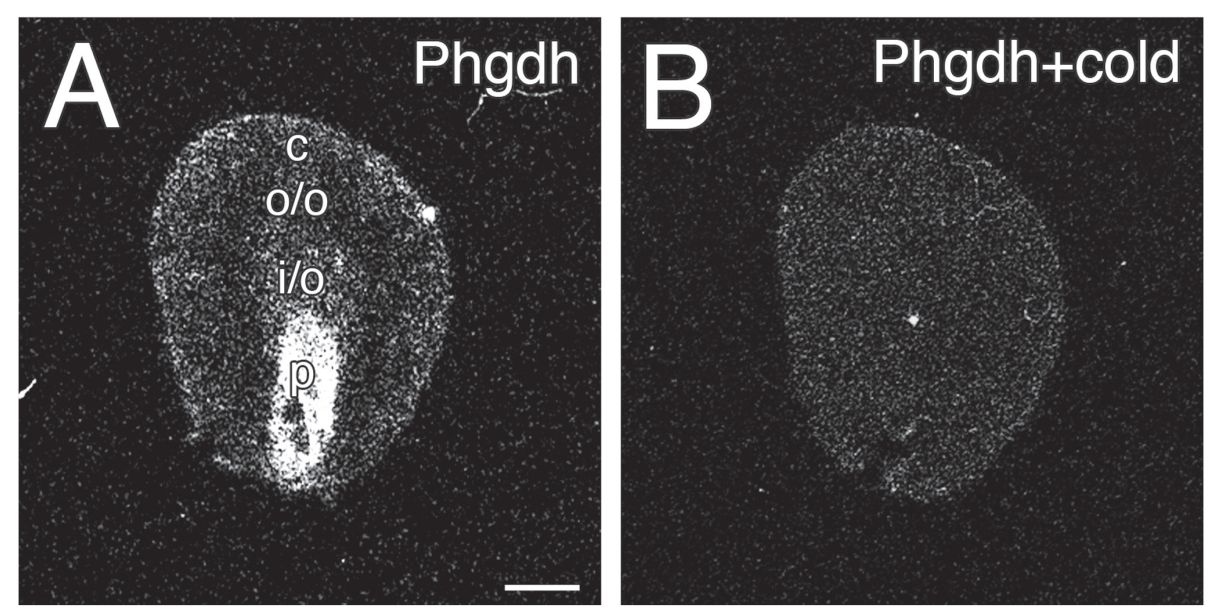

Fig. 1 In situ hybridization with X-ray film macroautoradiography showing expression for Phgdh mRNA in the adult mouse kidney. Hybridization was carried out in the absence (A) or presence (B, control hybridization) of an excess amount of cold (unlabeled) probes. c, renal cortex; o/o, outer layer of the outer zone; i/o, inner layer of the outer zone; $p$, renal papilla. Scale bar, $1 \mathrm{~mm}$. 


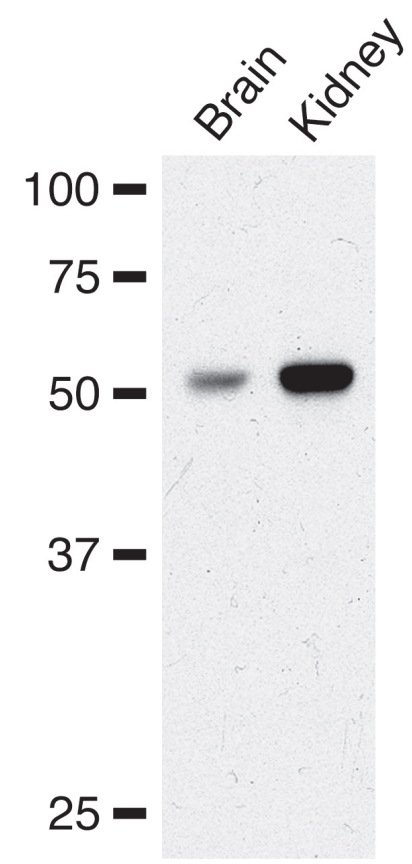

Fig. 2 Immunoblot with Phgdh antibody in the kidney (right) and brain (left). In both tissues, a single protein band at $60 \mathrm{kDa}$ is recognized. The position of standard protein markers is indicated to the left $(\mathrm{kDa})$.

dh was detected intensely in small-calibered renal tubules running through the renal papilla and inner layer of the outer zone (Fig. 3A, D-F), moderately in the Bowman's capsule (Fig. 3B), and weakly in certain renal tubules of the cortex (asterisk in Fig. 3B). Immunolabeling was low or negative in the outer layer of the outer zone (Fig. 3A, C). Thus, general distribution of Phgdh immunoreactivity was essentially the same as that of Phgdh mRNA, suggesting the specificity of immunohistochemistry. This was further supported by the lack of immunostaining when Phgdh antibody was replaced with normal rabbit serum (Fig. 3G, H).

Phgdh is expressed in Bowman's capsule, proximal tubules, and thin limb of Henle's loop

By multiple immunofluorescence labeling, expression of Phgdh in renal tubular segments was determined using segment-specific molecular markers (Fig. 4). Aquaporin-1 (AQP1) is a maker for the proximal tubule and thin descending limb of the Henle's loop (2). Phgdh was weakly detected in AQP1-positive proximal tubules withinin the cortex (arrows in Fig. 4A, B), and strongly in AQP1-positive thin-walled, small-calibered tubules, i.e., the thin descending limb of the Henle's loop (arrows in Fig. 4C, D). Double immunostaining for chloride

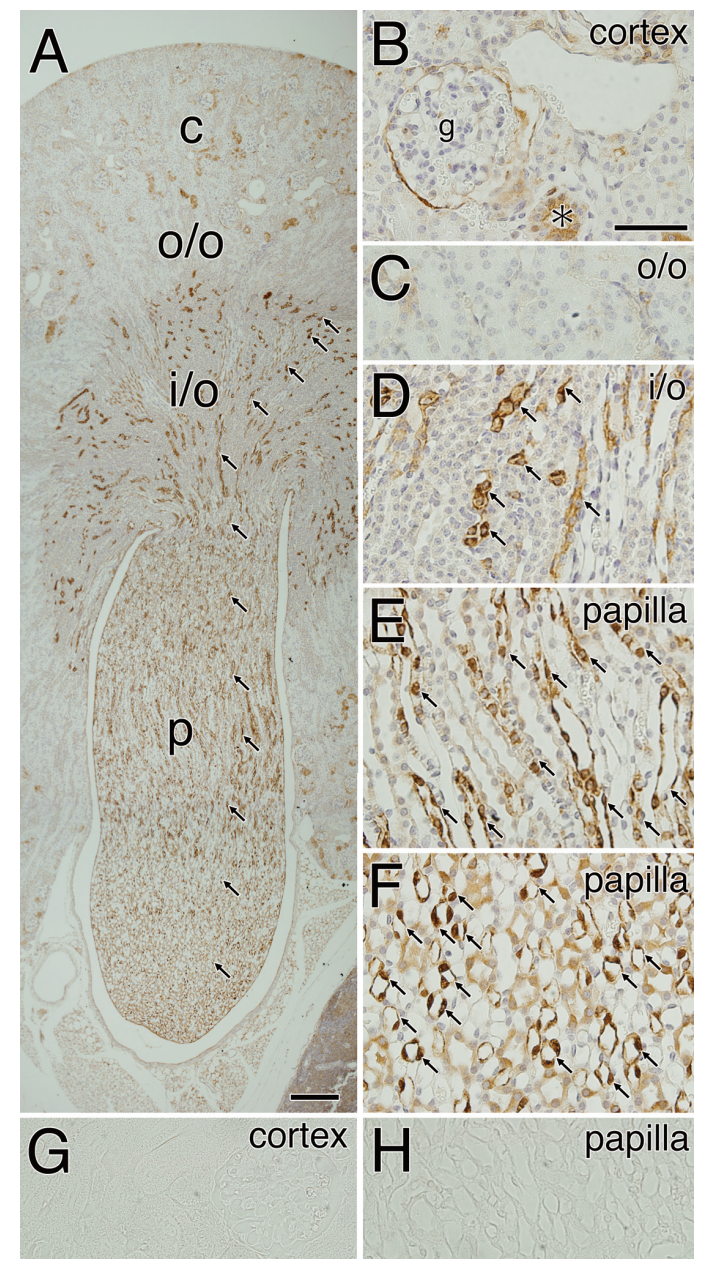

Fig. 3 Immunoperoxidase staining with rabbit Phgdh antibody $(A-F)$ or normal rabbit serum $(G, H)$ in the adult kidney. A, low-power view. $\mathrm{B}-\mathrm{H}$, high power views. An asterisk in Fig. $3 \mathrm{~B}$ indicates weak immunoreactivity in putative proximal tubules in the cortex. Arrows indicate intense immunoreactivity in the medulla. $\mathrm{g}$, glomerulus. For other abbreviations see Fig. 1. Scale bars, A, $200 \mu \mathrm{m}$; B (C-H), $50 \mu \mathrm{m}$.

channel $\mathrm{K}$ (CLC-K), a marker for the thin ascending limb of the Henle's loop $(21,26)$, demonstrated coexpression of Phgdh in this segment (arrows in Fig. 4E, F). In double immunofluorescence for calbindin, a marker of the distal convoluted tubule and connecting segment (20), and for aquaporin-2 (AQP2), a marker for the collecting duct (16), Phgdh was not detected in calbindin-positive distal convoluted tubules and connecting segment (arrowheads in Fig. 4G) or in AQP2-positive collecting ducts (arrowheads in Fig. 4H). Therefore, these results from light microscopic and confocal laser scanning microscopic immunohistochemistry indicate that Phgdh is expressed in the Bowman's capsule, proximal tu- 

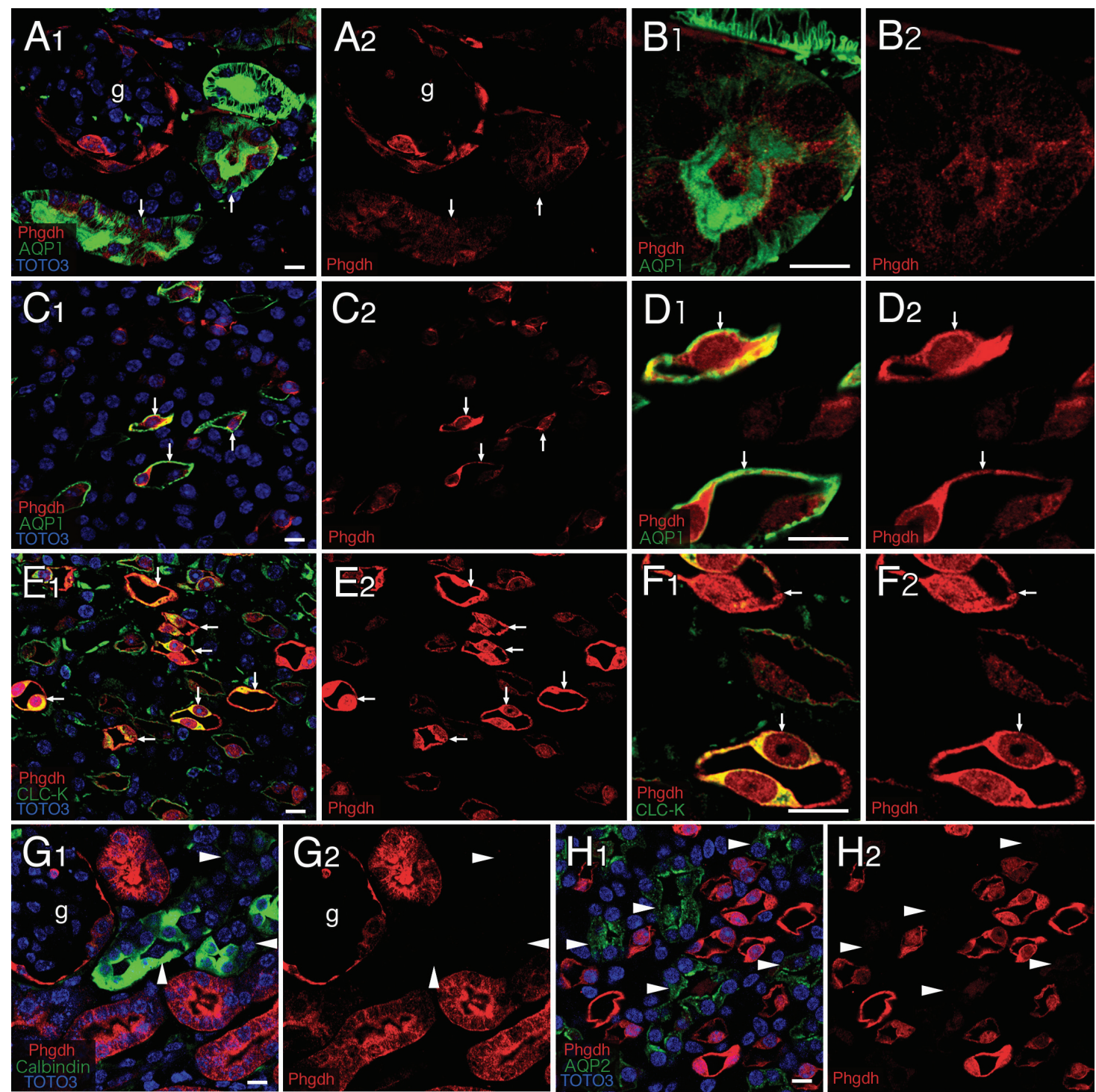

Fig. 4 Double immunofluorescence showing Phgdh (red) in Bowman's capsule, proximal tubules, and thin descending and ascending limbs of Henle's loop. A-D, AQP1 (green) for the proximal tubule (A, B) and thin descending limb of the Henle's loop (C, D). E, F, CLC-K (green) for the thin ascending limb of the Henle's loop. G, calbindin (green) for the distal convoluted tubules and connecting segment. H, AQP2 (green) for the collecting duct. Nuclear staining with TOTO3 (blue) is shown in merged images. Arrows indicate the tubular segments where Phgdh is overlapped with given segmental markers, whereas arrowheads indicate those where Phgdh is not overlapped with given segmental markers. g, glomerulus. Scale bars, $10 \mu \mathrm{m}$.

bule, and thin descending and ascending limbs of the Henle's loop.

This was confirmed by immunoelectron microscopy (Fig. 5). By the silver enhanced immunogold method, metal particles were deposited in the cytoplasm of squamous epithelial cells of the Bowman's capsule (Fig. 5A), and in that of epithelial cells of the proximal tubule (Fig. 5B). Among tubular segments, the heaviest labeling was observed in the cytoplasm of thin-walled, small-calibered epithelial cells of the Henle's loop (Fig. 5C). In contrast, labeling was very low or at the background level in epithelial cells of the distal tubule and collecting duct (Fig. 5D, E), consistent with light microscopic and confocal laser scanning microscopic observations (Figs. 3, 4).

\section{Phgdh and ASCT1 are expressed in distinct tubular} segments

Because L-serine is utilized for syntheses of various 

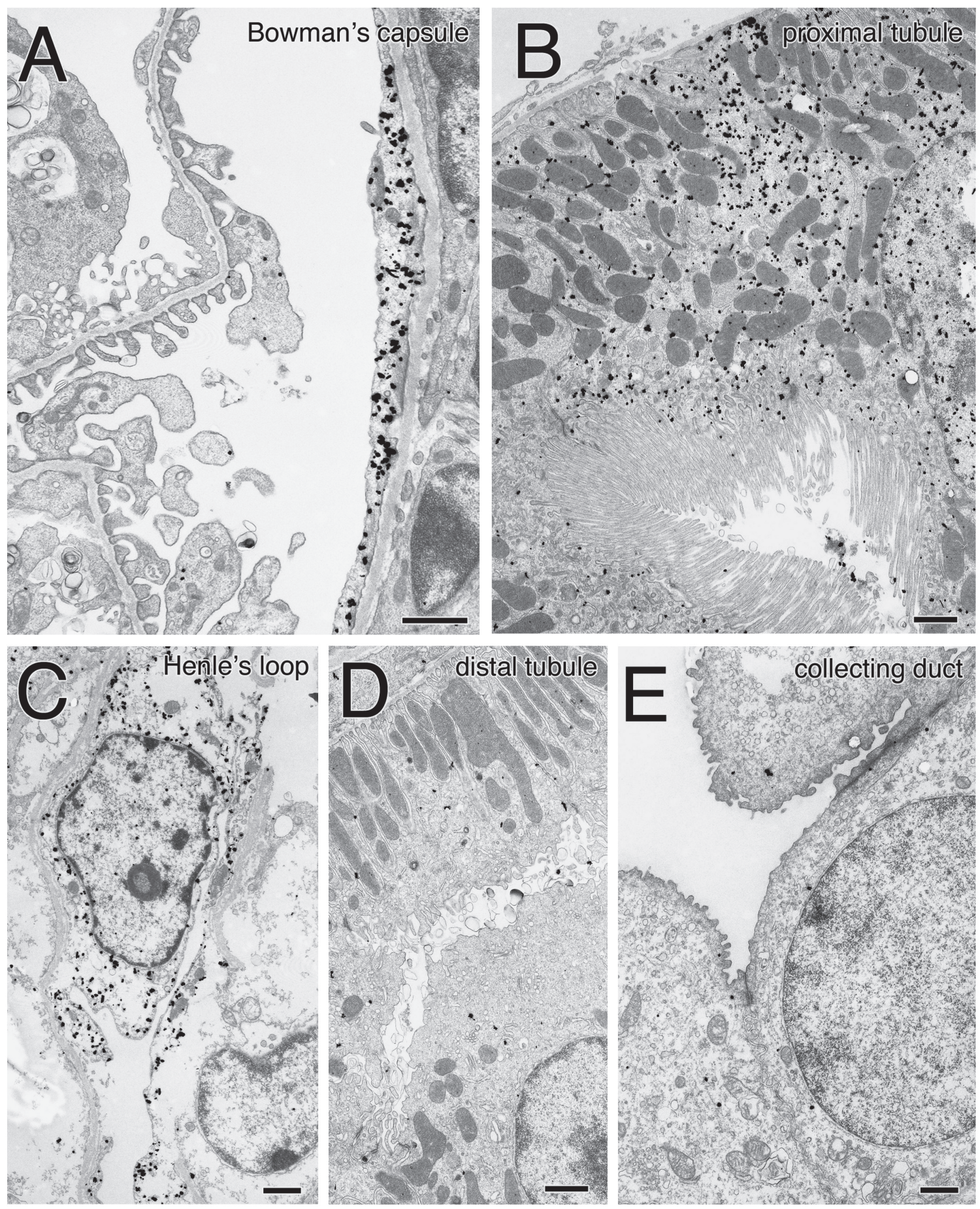

Fig. 5 Silver-enhanced immunogold electron microscopy for Phgdh in the renal cortex (A, B, D) and renal medulla (C, E). A, Phgdh is concentrated in the epithelial cell of Bowman's capsule. B, proximal tubule. Note the dense immunogold labeling in the cytoplasm and nucleus. C, thin limbs of Henle's loop. D, distal convoluted tubule. E, collecting duct. Scale bars, $1 \mu$ m.

Fig. 6 (Opposite page) Double immunofluorescence showing ASCT1 in distal convoluted tubules and collecting ducts. A, B, G, H, Phgdh (red) and ASCT1 (green). C, D, ASCT1 (red) and calbindin (green). E, F, K, ASCT1 (red) and AQP1 (green). I, J, ASCT1 (red) and AQP2 (green). L, ASCT1 (red) and CLC-K (green). Nuclear staining with TOTO3 (blue) is shown in merged images. Arrows indicate the tubular segments where Phgdh is overlapped with given segmental markers, whereas arrowheads indicate those where Phgdh is not overlapped with given segmental markers. Scale bars, $10 \mu \mathrm{m}$. 

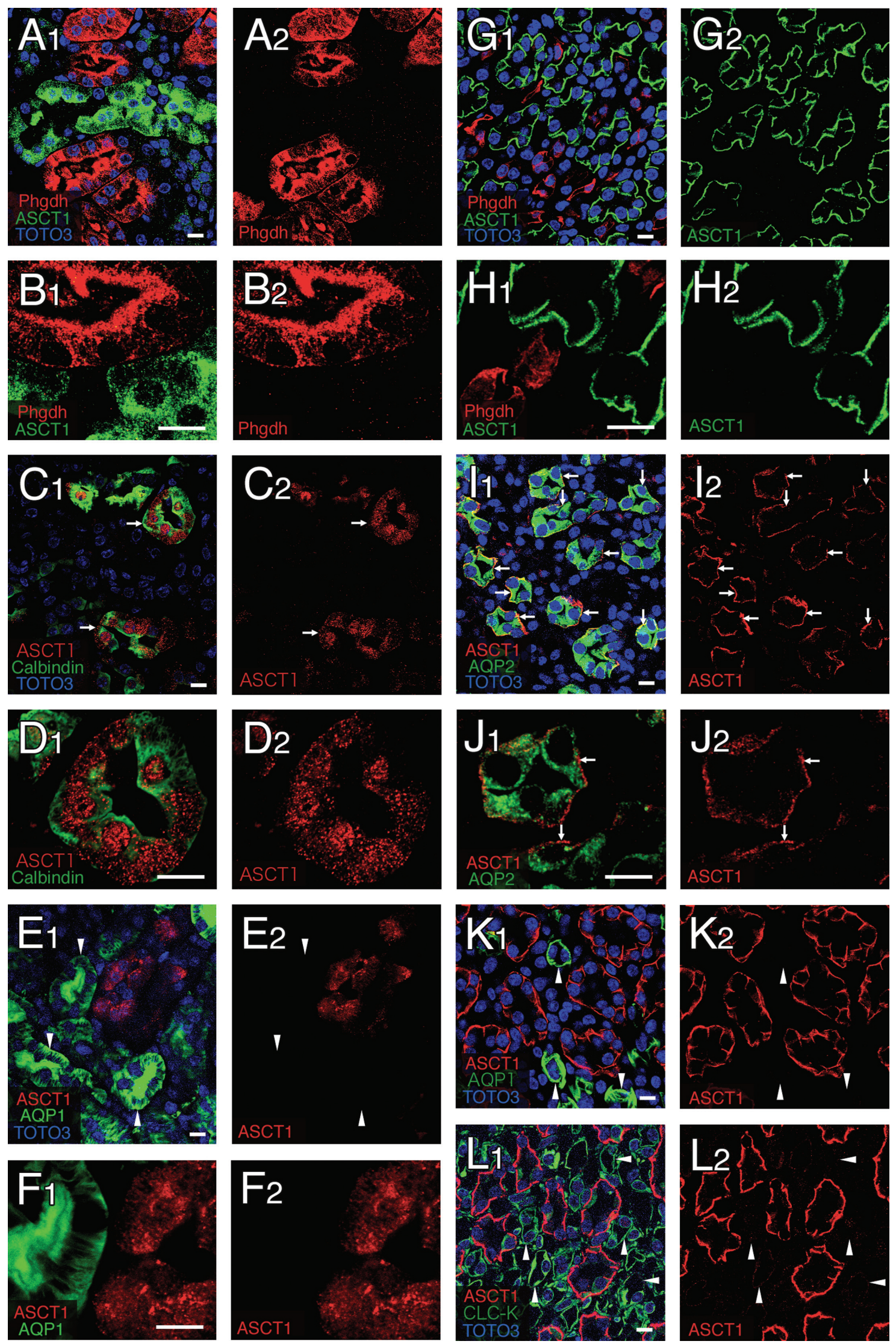
biomolecules, including other amino acids, proteins, membrane lipids, and nucleotides (10), renal cells lacking or low in Phgdh should somehow take in this amino acid for their activities, function, proliferation, and survival. To test this possibility, we finally examined the distribution of ASCT1 (Fig. 6), which is one of the system ASC or alanine/serine/ cysteine-preferring transporters $(3,18)$. Interestingly, we found non-overlapping patterns of Phgdh and ASCT1 expressions in the renal cortex (Fig. 6A, B) and medulla (Fig. 6G, H). In the cortex, ASCT1 was distributed in calbindin-positive distal convoluted tubules and/or connecting segment (arrows in Fig. 6C, D), but not in AQP1-positive proximal tubules (arrowheads in Fig. 6E, F). In the medulla, ASCT1 was detected in AQP2-positive collecting ducts (arrows in Fig. 6I, J), but not in AQP1-positive thin descending limb (arrowheads in Fig. 6K) or CLC-Kpositive thin ascending limb (arrowheads in Fig. 6L) of the Henle's loop. Therefore, Phgdh and ASCT1 display complementary expression in the kidney. Furthermore, we note different subcellular distribution of ASCT1 between the two immunopositive tubules. ASCT1 was detected widely in the distal convoluted tubule, irrespective of the apical and basal sides (Fig. 6A-D). In contrast, ASCT1 was preferentially distributed along the basolateral surface of the collecting duct (Fig. 6I-L).

\section{DISCUSSION}

In the present study, we analyzed L-serine biosynthetic enzyme Phgdh in the adult mouse kidney, since this organ is known to express its mRNA at high levels $(1,4,12)$. We detected a single protein band in the kidney, which is much higher in levels than that in the brain (Fig. 2). Even in the kidney with high tissue contents, cellular expression of Phgdh in the kidney was not ubiquitous, but rather specific to distinct tubular segments, i.e., the Bowman's capsule, proximal tubule, and thin limb of the Henle's loop. Together with our previous studies in nervous tissues $(9,22,24)$, the present result eventually highlights that, although the phosphorylated pathway of L-serine biosynthesis has been though to be a widely distributed cytosolic pathway $(11,19)$, the ability of L-serine biosynthesis is provided to particular cell types within given tissues and organs. In this regard, the fact that levels of Phgdh mRNA are highly variable from tissue to tissue (12) appears noteworthy, because this suggests that Phgdh is not a kind of housekeeping molecules and also because this could reflect cell type-specific expression in other tissues.

Why is Phgdh expression confined to distinct tubular segments? L-Serine is readily interconvertible to glycine, which is an important material as carbon and nitrogen donors for the purine and heme syntheses. In addition, L-serine is indispensable for the synthesis of phosphatidylserine and sphingolipids, i.e., basic components of the cell membrane and integral components of cell membrane microdomains, known as rafts and caveolae $(7,8,14,23)$. Therefore, one possibility is that tubular segments expressing Phgdh might be highly active in syntheses of proteins, nucleotides, heme, and cell membrane and thereby have especially high demands for L-serine than other segments lacking or low in Phgdh. Another possibility is that the different ability of L-serine synthesis would reflect different vulnerability to energy loss among renal cells, because L-serine is synthesized by depriving an intermediate of the glycolytic pathway, 3-phosphoglycerate. In the brain, it has been proposed that the lack of Phgdh in neurons would be beneficial for neurons themselves, because the availability of glia-borne L-serine may save their own glycolytic intermediates for preferential use in energy production, supporting neuronal survival and function (22). If this is the case in the kidney as well, tubular segments expressing Phgdh may play a role in the supply of L-serine to neighboring tubular segments negative in Phgdh expression.

In the present study, we found that expression of neutral amino acid transporter ASCT1 was also segmental within the kidney, i.e., the distal convoluted tubule and collecting duct. Importantly, this segmental expression was almost complementary to that of Phgdh. From the reciprocal pattern of expression, it becomes thus clear that each of renal tubular segments is provided with either biosynthetic enzyme or transporter for L-serine. Furthermore, the lack of ASCT1 in Phgdh-expressing tubules suggests that other transporters should be involved in the release or efflux of L-serine, if they play a role in L-serine supply to Phgdh-lacking tubules in the neighbor. In this regard, different subcellular distribution of ASCT1 between the distal convoluted tubule and collecting duct seems interesting. Wide intracellular distribution of ASCT1 in the distal convoluted tubule may represent L-serine uptake from the apical (or urine) and basolateral (or interstitial fluid) sides, while the preferential distribution in the basolateral side of the collecting duct may reflect its preferential uptake from the interstitial fluid.

This anatomical information will provide the ba- 
sis for future characterization of metabolic interaction and interplay mediated by non-essential amino acid L-serine in the kidney.

\section{Acknowledgement}

We thank to Drs. Toshihiko Iwanaga and Hiromi Takahashi-Iwanaga for helpful suggestion and discussion. This investigation was supported in part by Grant-in-Aids for Scientific Research on Priority Areas and for Scientific Research (B) from the Ministry of Education, Culture, Sports, Science, and Technology, Japan.

\section{REFERENCES}

1. Achouri Y, Rider MH, Schaftingen EV and Robbi M (1997) Cloning, sequencing and expression of rat liver 3-phosphoglycerate dehydrogenase. Biochem $J$ 323, 365-370.

2. Agre P (2000) Aquaporin water channels in kidney. $J \mathrm{Am}$ Soc Nephrol 11, 764-777.

3. Arriza JL, Kavanaugh MP, Fairman WA, Wu YN, Murdoch GH, North RA and Amara SG (1993) Cloning and expression of a human neutral amino acid transporter with structural similarity to the glutamate transporter gene family. $J$ Biol Chem 268, 15329-15332.

4. Cho HM, Jun DY, Bae MA, Ahn JD, and Kim YH (2000) Nucleotide sequence and differential expression of the human 3-phosphoglycerate dehydrogenase gene. Gene 245, 193-201.

5. Davis JL and Fallon HJ (1970) Studies on the role of 3-phosphoglycerate dehydrogenase in the regulation of serine biosynthesis in rat liver. $J$ Biol Chem 245, 5838-5846.

6. de Koning TJ, Snell K, Duran M, Berger R, Poll-The BT and Surtees R (2003) L-serine in disease and development. Biochem $J$ 371, 653-661.

7. dos Santos Fagundes I, Rotta LN, Schweigert ID, Valle SC, de Oliveira KR, Huth Kruger A, Souza KB, Souza DO and Perry ML (2001) Glycine, serine, and leucine metabolism in different regions of rat central nervous system. Neurochem Res 26, 245-249.

8. Furuya S, Ono K and Hirabayashi Y (1995) Sphingolipid biosynthesis is necessary for dendrite growth and survival of cerebellar Purkinje cells in culture. J Neurochem 65, 15511561.

9. Furuya S, Tabata T, Mitoma J, Yamada K, Yamasaki M, Makino A, Yamamoto T, Watanabe M, Kano M and Hirabayashi Y (2000) L-serine and glycine serve as major astroglia-derived trophic factors for cerebellar Purkinje neurons. Proc Natl Acad Sci USA 97, 11528-11533.

10. Furuya $S$ and Watanabe M (2003) Novel neuroglial and glioglial relationships mediated by L-serine metabolism. Arch Histol Cytol 66, 109-121.

11. Ichihara A and Greenberg DM (1957) Studies on the purification and properties of D-glyceric acid kinase of liver. $J$ Biol Chem 225, 949-958.

12. Klomp LW, de Koning TJ, Malingre HE, van Beurden EA, Brink M, Opdam FL, Duran M, Jaeken J, Pineda M, Van Maldergem L, Poll-The BT, van den Berg IE and Berger R (2000) Molecular characterization of 3-phosphoglycerate dehydrogenase deficiency - a neurometabolic disorder associated with reduced L-serine biosynthesis. Am J Hum Genet 67, 1389-1399.
13. Mitoma J, Furuya S and Hirabayashi Y (1998) A novel metabolic communication between neurons and astrocytes: nonessential amino acid L-serine released from astrocytes is essential for developing hippocampal neurons. Neurosci Res 30, 195-199.

14. Mitoma J, Ito M, Furuya S and Hirabayashi Y (1998) Bipotential roles of ceramide in the growth of hippocampal neurons: promotion of cell survival and dendritic outgrowth in dose- and developmental stage-dependent manners. J Neurosci Res 51, 712-722.

15. Miura E, Iijima T, Yuzaki M and Watanabe M (2006) Distinct expression of Cbln family mRNAs in developing and adult mouse brains. Eur J Neurosci 24, 750-760.

16. Nielsen S, DiGiovanni SR, Christensen EI, Knepper MA and Harris HW (1993) Cellular and subcellular immunolocalization of vasopressin-regulated water channel in rat kidney. Proc Natl Acad Sci USA 90, 11663-11667.

17. Sakai K, Shimizu H, Koike T, Furuya S and Watanabe M (2003) Neutral amino acid transporter ASCT1 is preferentially expressed in L-Ser-synthetic/storing glial cells in the mouse brain with transient expression in developing capillaries. J Neurosci 23, 550-560.

18. Shafqat S, Tamarappoo BK, Kilberg MS, Puranam RS, McNamara JO, Guadano-Ferraz A and Fremeau RT Jr (1993) Cloning and expression of a novel $\mathrm{Na}^{+}$-dependent neutral amino acid transporter structurally related to mammalian $\mathrm{Na}^{+} /$glutamate cotransporters. J Biol Chem 268, 1535115355.

19. Snell K (1984) Enzymes of serine metabolism in normal, developing and neoplastic rat tissues. Adv Enzyme Regul 22, 325-400.

20. Taylor AN, McIntosh JE, Bourdeau JE (1982) Immunocytochemical localization of vitamin D-dependent calcium-binding protein in renal tubules of rabbit, rat, and chick. Kidney Int 21, 765-773.

21. Vandewalle A, Cluzeaud F, Bens M, Kieferle S, Steinmeyer $\mathrm{K}$ and Jentsch TJ (1997) Localization and induction by dehydration of CLC-K chloride channels in the rat kidney. $\mathrm{Am} \mathrm{J}$ Physiol 272, F678-F688.

22. Yamasaki M, Yamada K, Furuya S, Mitoma J, Hirabayashi Y and Watanabe M (2001) 3-Phosphoglycerate dehydrogenase, a key enzyme for L-serine biosynthesis, is preferentially expressed in the radial glia/astrocyte lineage and olfactory ensheathing glia in the mouse brain. $J$ Neurosci 21, 7691-7704.

23. Yamashita T, Wada R, Sasaki T, Deng C, Bierfreund U, Sandhoff K and Proia RL (1999) A vital role for glycosphingolipid synthesis during development and differentiation. Proc Natl Acad Sci USA 96, 9142-9147.

24. Yamashita N, Sakai K, Furuya S and Watanabe M (2003) Selective expression of L-serine synthetic enzyme 3PGDH in Schwann cells, perineuronal glia, and endoneurial fibroblasts along rat sciatic nerves and its upregulation after crush injury. Arch Histol Cytol 66, 429-436.

25. Yoshida K, Furuya S, Osuka S, Mitoma J, Shinoda Y, Watanabe M, Azuma N, Tanaka H, Hashikawa T, Itohara S and Hirabayashi Y (2004) Targeted disruption of the mouse 3 -phosphoglycerate dehydrogenase gene causes severe neurodevelopmental defects and results in embryonic lethality. $J$ Biol Chem 279, 3573-3577.

26. Yoshikawa M, Uchida S, Yamauchi A, Miyai A, Tanaka Y, Sasaki S and Marumo F (1999) Localization of rat CLC-K2 chloride channel mRNA in the kidney. Am J Physiol 276, F552-F558. 
